\title{
From the quantum to the macroscopic level: a new systemic model for our complex system
}

\author{
Sara Diani, M.D. \\ Université Européenne Jean Monnet, "Giovanni Ferrari" School of Specialization in Music \\ Therapy, Padua, Italy \\ dr.saradiani@gmail.com
}

ORCID: 0000-0003-3079-0325

\begin{abstract}
As a Complex System, our body acts as a whole system connected to the environmental incitements. It is ordered, coherent, tries to maintain the least possible entropy, saving the greatest amount of energy. We can observe its active systemic response to environmental information both when it is healthy and ill. To explain the dynamics of the systemic regulative network a theoretical model is proposed, with a comprehensive approach that allows seeing the entire regulative system as a continuous unicuum. The paper analyzes two points of view: 1) the connections between the quantum level and the classical one, through some principles of the QFT and through the Coherence Domains. The system is modeled as a field described by the wave function, with synchronous and consistent events, driven in a global computing by the quantum potential Q. The quantum potential implies the nonlocality, and it needs only ultra-weak waves to occur, so it explains how the rapid and global activation of the organism in response to punctiform information work. The initial hypothesis is that some consistent quantum phenomena are amplified through the systemic regulative network until they become macroscopic observable. This is possible because of Coherence Domains. 2) The reactions of the different systemic networks to perturbations/ punctiform information, with the first attempt to model and measure information in biology, going beyond the Shannon and Turing theories. Hopfield Networks and an informational point of view are used to address the crucial informational and organizational role of proteins and nucleic acids. With this new frame we could develop innovative therapeutic strategies, and also evolve new experimental way to make our clinical observation more precise.
\end{abstract}

Keywords: Quantum Network, Quantum Potential, Complex System, Coherence Domains, Hopfield Networks, Information 


\section{Introduction}

2. The two new premises and considerations

3. Applied Quantum Field Theory

4. Coherence Domains

5. How proteins act: Hopfield Networks

6. The informational point of view

7. From the quantum to the classical level - the macroscopic observation

8. Conclusion

\section{Introduction}

We are a Complex System (CS). As a CS, our body acts as a whole system connected to the environmental incitements. It does so in an orderly, coherent way, with the least possible entropy, and saving the greatest amount of energy. We can observe its active systemic response to environmental information both when it's healthy and ill [1].

The question is: at what level do we enter this accuracy in the systemic response, this consistency, this energy saving and great susceptibility we have? I suggest the presence of a new level of singular and systemic activity, deeper than the normal observed physiology. More depth is required, especially to understand how our body self-regulates itself, how it organizes its processes and information that it receives and exchanges.

This is the first model that combines classical physics and all that is observable in our body, the reaction to the puctiform information, and the implementation of an actual reaction through a network that is activated and expresses properties of quantum physics, in particular the quantum field, through a consistent behavior and organization. In this sense, the model integrates all concepts related to dissipative complex systems far from thermodynamic equilibrium $[2,3]$.

I use a systemic, monistic vision and consider our body and mind as a whole entity. It is rightly so, being the complex system actually indivisible.

There are many physical models that explain the central nervous system, consciousness and brain cognition from the logic and quantum point of view (for example [4-7], or the work of Freeman and Vitiello [8-10]). I move even further, arguing that the ability to learn, to process incitements and consequently develop a cognitive and intelligent response is characteristic of the whole organism; we are therefore required to have a unified view [11, $12]$.

This is why this theoretical model is intended to be systemic. It analyzes two points of view: 1) the connections between the quantum level and the classical one, through some principles of the QFT and through the Coherence Domains; 2) the reactions of the different systemic networks to perturbations/punctiform information, with the first attempt to model and measure information in biology, going beyond the Shannon and Turing theories. 


\section{The two new premises and considerations}

There are two premises, two fundamental assumptions:

1) The first hypothesis is the following: there is a network control system, global and ordered, that underlies the processes we observe, that we don't normally consider in our pharmacological and clinical studies. This allows implementing the response network in a systemic and comprehensive way, as if the system were a unit. This network is deeper, of orders of magnitude lower than those we normally observe in physiology and pathophysiology studies. The network is sensitive to information that initially does not come in contact with the receptors; they do not activate them directly. This means that, in addition to knowledge of the environmental and internal stimuli through the receptor, there is a deeper system of knowledge, which initially does not require a receptor, but micro-changes in the organic field. These small molecular fluxes will activate the receptors. These incisive movements occur at particular points, the network nodes, which influence it consequently.

This is consistent with the operation of a complex system: it is, by definition, susceptible to perturbations/punctiform information at the boundaries, causing a cascade of events that spreads according to the rules of diffusion, amplification and compartmentalization of the organism.

It is possible to imagine such susceptibility: for example there are photosensitive molecules, such as hemoglobin, which are sensitive to light without having receptors.

The premise is that a quantum network can describe a body better, more accurately, in a contextual and holistic way. This approach is therefore essential, because the goal of medicine is to understand and describe the uniqueness of the system by studying the history of its specific responses.

It is from this regulative network of control that non-deterministic behavior arises: these are the point of deterministic chaos. The dynamic regulations between sub-systems do not work in a deterministic way, and this because they interfere with quantum events. This is the mastermind of the systemic changes which we observe.

Understanding how this network works, at what level is, what kind of sensitiveness and precision it has, and how to stimulate it to achieve the desired systemic effect is the big step that this model will allow to achieve.

If we had this ability, we could stimulate the system according to its laws, so that it would regulate itself deeply and precisely, not coarsely. We would not need to lock any of the current processes, as we could just support the system in adjusting itself. We would not have side effects, or we would have very few, because our information would be punctiform, precise, and would impact only the points of the network that are susceptible, in a delicately way. The answer we would observe would be consistent, fast (at least in the initial 
triggering), concerning the organism as a whole, and we would see how the response networks can activate and influence each other, simultaneously. It would be revolutionary. We could stop locking or suppressing it, increasing the systemic entropy, providing just an ephemeral and temporary relief from suffering in most cases.

I will use in equivalent manner the following terms: control network, network of downregulation, regulation system, quantum regulation system or regulatory network.

2) When we administer a weight drug which influences the receptors, we act on the physical network of the system, i.e. on the surface, not on the deep intelligent one. As a result we see only the effects filtered by the receptors, and we don't consider the primary changes on the regulatory network.

Every drug, every substance ingested by the body, has two components: a physical one, weight, described by the properties of classical physics, biochemistry, biology and so on, and an informative, described by the physics of information. Consequently, every drug has two types of effects: using the Hahnemann nomenclature [13] the primary effects are those of the administered substance that the system undergoes at the beginning; secondary effects come from the receptor opening and all the consequent active actions. These are proper of the body to which the remedy is given. If we give only one weight stimulus, its primary effects are hidden from the secondary ones, occurring simultaneously. I do a prediction: if we would be able to send punctiform information to the body in the form of ultra-weak, precise waves, which acts primarily on the regulation network, we would observe before the primary effects, generalized and that can be expressed in all their variety; then, only later, secundary and side effects, typical of the systemic reaction.

It is necessary to specify the following things: there is currently no clear point of the boundary between quantum and classical physics [14]; it was understood only recently that the senses of sight, smell, the ability to navigate through the magnetism of some animal species and photosynthesis are connected to basic quantum phenomena [15]. All the biological structures can be described in biochemical terms, and we can go deep into analyzing and observing the processes at the level of ions, proteins, small molecules. This is the level where the behavior relies on the rules of quantum physics.

In addition, the largest object with which quantum was tested weighs $10^{5} \mathrm{amu}$, while the smallest object to test the classical physics weighs $10^{18} \mathrm{amu}\left(10^{-6}\right.$ grams $)$. Between these two values, there is the unknown [16]. Singh proposes to divide the regions of space in microregions, where quantum rules over gravity, transition regions and macro-regions, where gravity and classical physics seem to dominate on quantum.

In addition to this uncertainty, there is a big problem for those who want to use quantum models to describe our body and its functioning: the quantum decoherence.

There is another issue: the high incidence of the observer's role. The observation has the effect of modifying reality. We, as observers, change the relationship between the reliability and independence of information obtained by the system [17]. This, besides the fact that the measurement disappears with the overlap of the quantum states, causes information loss 
[18]. While measuring, the quantum state becomes bound (entangled) with a part of the universe, for example with the measurement tool [19].

The implications concerning the observation of system's activities are mainly two:

1) The wave function of the observer must collapse, in order to allow the observation of the process.

2) The system will auto-observe, producing at each system self-observation a collapse of the wave function $\mathrm{R}$. This is a concept that should be expanded, since we do not know the ways in which this mechanism is applied.

The relationship between observer and observed is an adaptive process in which the system's internal logic meets our mode of acquiring information [20].

It's hard to think of $U$ (the function of quantum coherence) and $\mathrm{R}$ as two alternating processes and never contemporaries, because this implies an elaboration of reality which is discontinuous and discrete anyway.

There are some theories that seek to reconcile $U$ and $R[16,21-26]$. This is a central issue which I will not deal with at length in this model. The only idea I would like to affirm now is that there are continuous transitions where $\mathrm{U}$ and $\mathrm{R}$ occur simultaneously.

There are always more physiological processes described precisely by quantum physics, as suggested by Tarlaci and Pregnolato [15]. The task of quantum biology is to identify as much as possible and expand this knowledge. Moreover, ion channels, protein, and also the DNA breakage due to ultraviolet waves, as well as perhaps the consciousness, depend on phenomena that can be described with quantum equations.

\section{Applied Quantum Field Theory}

The theory that fits the best with the model describing our reaction to punctiform information is the quantum field theory (QFT). It solves four issues raised by quantum mechanics:

- The wave-particle duality.

- The inability to dynamically create or destroy the particles, which is a crucial aspect of relativity. These processes must be explained and considered in a truly relativistic quantum theory.

- The problems that occur when trying to apply the Schrödinger equation for large particle numbers.

- The need to explain the non-locality, that is, the interaction of seemingly independent particles even at a distance, with instantaneous changes of their properties (for example spin).

For these reasons Dirac proposed the second quantization. The field is a continuous entity that exists in every point of space and regulates the creation and annihilation of particles. Even the quantum field has a Hamiltonian H (more complex than typical Hamiltonians of 
individual particles), and obeys Schrödinger equation, which is divided by Bohm theory [27, 28] in a real part and an imaginary part.

$$
Q=-\frac{\hbar^{2}}{2 m} \frac{\nabla^{2} R}{R}
$$

So we get the quantum potential Q. According to this new interpretation, the electron does not occur randomly, but moves according to the action of the quantum potential. Q carries information of the global environment and the field through non-local connections, which occur in a simultaneous and synchronous manner. Synchronicity, causality, non-locality and the potential determinability of the electron trajectory are the fulcrums of the QFT. We still cannot predict the electron's behavior from our poor knowledge of the initial conditions, and the continuously changing nature of the field. The QFT no longer considers the wave-particle duality, but is a good network of impacts, also said interaction vertices, in which some properties (such as the position, the spin), are constantly being created and destroyed. The properties that we measure are emerging with respect to the events' network [29].

The single electron has a field which becomes the field of all the electrons. There are overlaps of states, from which the particles emerge. Then the particles are not "persistent object", but networks of energy transitions. The wave-particle language is only an approximate language, and the world is made up of this dynamic network of energetic phenomena. The non-locality is thus a key ingredient of QFT.

These overlays given by the non-locality, together with the fact that the particles are not more persistent objects but are summations of distant events linked together, make the individual changes intelligible. Associative quantum networks map the structures and produce interference. They are an implementation of the classical Hopfield networks. Information is the statistical sum of what happens at the quantum level [30]. In practice, the quantum potential brings global information about the process and its environment. It is an active source of information, connected with the quantum background (implicit information carrier) that acts in the space-time in which measurements, i.e. the explicit quantum information, are made. With our measurements we make a selection of information which follows an implicit order. This is crucial for the understanding of our body, because in this way we think we can observe events otherwise inaccessible.

The particle is more sensitive to the wave shape than to its intensity, so the effect of $Q$ cannot vanish where the wave function amplitude tends to zero. Another key feature is that the quantum potential acts even at a distance, with effects that depend on the wave function. Theoretically, only uniform rectilinear motions should be possible because, according to classical mechanics, they are the only ones that do not take place in the presence of forces. These are two key points.

For these reasons I use the QFT as a key theory for a model of control system which 
underlies the phenomena we observe.

To summarize the points discussed above, and begin to model the body concretely, it is stated that:

- The system is described by a field which propagates in space and time, and from a punctiform structure, the identity, which moves under the guidance of the field.

- The field is described by the mathematical point of view by the wave function which is the solution of the Schrödinger equation.

- It is this quantum comprehensive approach that allows us to model the entire body regulation network as a continuous unicuum.

- In the field, events occur synchronously and consistently. This is critical and explains for example some hyper-acute global reactions.

- The quantum potential is the variable that is indicative of the changes that occur in the system, and that drives the events. It allows us to consider the non-locality and acts at distance, needing only ultra-weak waves to occur. Also it synchronizes all the individual processes in a global computing. This entity explains how the rapid and global activation of the organism in response to a punctiform information is possible.

- In classical physics, a particle cannot exceed a potential barrier $\mathrm{V}$ if its kinetic energy is less than the height of the barrier. In QFT instead, because of the probability distribution, sometimes the particle will overcome that barrier [31]. In fact, when a set of wave functions comes in contact with it, very rapid changes of the quantum potential $Q$ take place, which may become negative, lowering the threshold of potential $\mathrm{V}$ and allowing the passage of the particle. So the ability to cross the barrier is given by the sum of $\mathrm{V}+\mathrm{Q}$. Of course the kinetic energy of the particle must be greater to $\mathrm{V}+\mathrm{Q}$. In our case the barrier can be an ion channel, for example.

The fact that it activates in response to events that would not exceed the activation threshold is critical and explains the increased susceptibility of the organism.

- Even the observer's system is described by a wave function. When the two systems (observed and observer) interact, takes place a sum of their wave functions, for which the quantum event of the system is the element of the physical reality of the observer.

- The observer will not be able to observe the system in a deterministic manner and using the computational and informational model Shannon-Turing. This although the system openness.

- Events and all the possible occurrences have in the system certain probability density. The observer, through the reduction $\mathrm{R}$ of the state vector, will derive its properties.

- Licata [20] suggests that the separate Hilbert spaces are not suitable for the QFT. Not separated Hilbert spaces are necessary. We could find in this concept an analogy with the logic of the inseparability of complex system compartments.

- If we do give any precise and punctiform information to the system, this will be in the form of a ultra-weak wave. Therefore its wave function is added to that system. The results that can arise are the appearance of new symptoms/observables or the resolution of those 
already present.

This ultra-weak wave must be consistent with the system, have a similar wave function and be afferent to its linguistic domain. The linguistic domain corresponds to the wave function of the system and to all states (more or less likely) that it can take. The wave that we dispense must be specific and individualized; it must have an informed dissipative structure, as our body does. Whether it is effective or not depends from its energy, the difference between its energy and that of the system, the state of the system at that time, and the consistency between the phases of the system and those of the remedy. The quantum network will be affected by this wave, and will change accordingly.

From these quantum events some externally observable ones will emerge, and what I am about to explain is the bridge between quantum physics and classical physics. This is the big and decisive step we need, and that I discuss further.

\section{Coherence Domains}

The part of the model that follows explains the primary effect of a substance, or the effect due to the information itself, which affects the body.

The premise is that the information does not directly activate the receptors.

In fact it is the concentration or the location of the micro molecules or ions present in the water which is initially locally modified (the body of the newborn is composed of $75 \%-85 \%$ water, that of the adult by $70 \%$, up to $60 \%$ for the elders). The water of which we are composed is a set of ions, solutes, proteins, and is compartmentalized, divided between the extracellular space and intracellular space.

We must rely on the coherence domains of Del Giudice [32]. The Italian physician in fact has hypothesized and demonstrated that the water present in the living matter is not inert, but is organized in coherence domains that behave like dipoles, and that in this excited state oscillate between two states with different spatial configurations. They contain water, ions and proteins. These trap the quantum potential, so that it will use its influence on every charge or polarizable molecule that is found in these domains [33]. In practice, the electromagnetic field is trapped from atoms and molecules and oscillates in phase with them, from rest to excited state. This ensures consistent evolution to the system, and acts as glue between its sub-components. The coherence domains are of the order of magnitude of tens of microns (the size of a human cell may vary from 10 to 100 microns, with a few exceptions); their main function is to interact with weak electromagnetic signals, and store the information that they carry. There is a vector that also expands over the system, and exchange information only with the environment in which it is immersed, without energy. It allows then for continuous and subtle communication, which can be observed only from the wave point of view. Another important thing is that the entropy within the domain is zero. Living matter is the simultaneous coexistence of coherence and decoherence. The 
inconsistent molecules move according to a selective recall, following the code between the resonant frequencies of the coherence domains. Later on they create the membranes which are consistent, and that then attract other molecules. In this way they change the phases and the oscillation modes of the involved fields. The quantum potential correlates with each other all these facilities and these phenomena. Therefore fluids are not organized as a whole in coherence domains, but there are contemporary inconsistent set of molecules and selforganized ones in compartmentalized domains. It is logical to think that the healthier the body is, the more diffuse and widespread the organization is. In this case there are more water molecules bound to the protein, and consequently the organism is more susceptible to ultra-weak specific waves. With the disease, it increases the ratio of free water compared to that bound to macromolecules. With the decreased presence of coherence domains, there will be on one hand a less harmonic, consistent and specific response, and on the other a lower susceptibility to specific information. This may be the reason why children usually respond better to the treatment than the elderly. So it is much more difficult, if not impossible, to cure an advanced disease. Where the consistency decreases, increases the entropy. This concept of water more or less bound to macromolecules is already used in MRI, in which the T1 signal measurement time is shorter if the water is more closely linked to macromolecules, and so the image is clearer. With more free water, T1 is longer and the image of the tissue appears hypo-intense, so darker. In this way normal tissues are distinguished from pathological ones.

According to Del Giudice, the living system is the final stage of a dynamic evolution which arises from the basic interactions within a set of electric dipoles, where the dipole is a schematic representation of a biomolecule [34]. We can consider the oscillation of the dipole as a swing product of its pair of electrons. Obviously the dipoles are not isolated. They are in chains, and each linked to the other. The dipoles may interact with each other via shortrange Van derWaals forces and hydrogen bonds, and long-range electromagnetic force (Coulomb and radiative).

The energy of the information we provide is equal to:

$$
\mathrm{E}=\mathrm{hv}=\mathrm{hc} / \lambda
$$

This field to be effective needs a wavelength $\lambda$ similar to that of the coherence domain, and a density $\mathrm{N} / \mathrm{V}$ (Number of atoms/Volume in which the system is contained) that exceeds the critical threshold. So it will resonate with our system, and take the transition to a new phase. The electromagnetic field that we have introduced is now trapped in the coherence domain, and a sum of their wave functions actually happens. If they are in the same stage, the previous pathological wave function will also be annihilated by subtraction.

That is what we want when we try to treat the root of a disease: we want the network of symptoms to extinguish itself, without blocking anything. To do this, it is necessary that the quantum control system no longer expresses its pathological wave characteristics. The 
pathological wave should then gradually and spontaneously extinguish itself. Only if we act at this basic level we can get healthy and lasting changes. If we instead block some partial pathological aspect in the most superficial level, like what we are actually doing, the adjustment will rally the network; it will give rise to a new response likely to be more disorganized than the last, with more entropy and energy expenditure. Probably some symptoms will disappear, but after some time they will manifest again. After an initial period of decrease or disappearance of symptoms, these will return with increased intensity. So, back to the model, the water is coupled with solutes of various nature, including proteins, and acts in a consistent and informed way. Now I will reflect on the role of the proteins compared to the dipole. The proteins, with their shape, their folding and their active sites, change the water polarization, and mate to it structurally in coherence domains. They behave as a set of spin vitrified networks, or Hopfield networks. When the water is stimulated, the dipole acts as a switch, wherein the protein can be activated through its active sites, and activating other.

\section{How proteins act: Hopfield Networks}

The vitrified spin networks were introduced by Hopfield [35, 36], they are neural networks. Always with the same previous argument, we argue that the ability of the organism to obtain information and to act cognitively implies that some properties of neurons and their networks are similar to those which occur throughout the body, and are then explained by this model.

These neurons have $\mathrm{N}$ inputs, and also have an output that is continually updated. Each input $i$ has an associated weight $w_{i}$, and all weighted inputs are summed. The output will be +1 if their sum will be greater than or equal to zero, and will be -1 if the sum will be less than zero. All the neurons are interconnected, and also here each connection is weighed and inserted into a matrix.

These networks organize themselves, without external organizers, forming maps and accumulating memories [37]. The mutual effects between adjacent elements, which take place on the basis of local interactions, generate the global schematization. That is why they are complementary to the Coherence Domains, and useful to this model. In the Hopfield networks it has been observed that by changing one or two elements, these do not return to their initial state, but introduce a previous balance distortion, which is then transmitted from one component to another, and so forming a new balance, ready to be disturbed again by a new weight input and specificity sufficient to cause an output. The new provision in a sense constitutes the network memory. Balance is in fact ephemeral, so that the network elements are called frustrated because the spins and the layout are always ready to change. Again it is emphasized the dynamism of these plastic structures. But in fact, every state reached after a stimulus is a pseudo-stable state of minimum energy; we 
could say a behavioral attractor. There are compartments that define interconnected but partly independent fields, with an increased susceptibility to changes at their boundary. Furthermore, the other analogy with the coherence domains is that if the network elements are consistent with each other, they reinforce each other, and then amplify the change.

I can state that an ultra-weak and ultra-specific wave affects one or more domains of coherence, or edits in a punctiform way the network field down-regulation, which is sensitive to this type of information. The proteins play the most important role, because of their folding, of their active sites and the capacity to accumulate and manage information, begin to swing together with their entire coherence domain. We can represent the oscillations of the water dipole as binary states 0 or 1 , which then behave as switches. From the initial change of state in a domain, or in a part of the network field, influence originates towards compartments and domain neighbors, and activates a cascade of events. This has two directions: on one side protein networks are activated, in ways that vary according to the type of information; this is the primary effect. On the other hand, these changes of coherence domains cause micro flows of ions and molecules, which may increase the scope and reach the cell receptors. These receptors open up, become activated and trigger the cellular response; from this springs the secondary effect, with the opening of new networks and the production of new molecules from cellular DNA. Although the cells are organized in coherence domains, this means that they can act also on the peri-cellular field. This gives a very rapid response, overall synchronous to each level. By acting on this system of regulation the effects throughout the body are obtained, although not all will become observable. This allows a modulation that is much more versatile, precise and harmonic.

The information core that through this network takes root and acts is the essence of life.

The quantum potential is the director, and the laws of quantum field allow these great evolutions from stimuli that normally would not exceed the classical activation threshold.

So the coherence domains can amplify the initial ultra-weak wave through the regulative network, and after that to the more superficial level. It is because of this organization and activity that the quantum phenomena do not nullify each other, but some of those emerge and become macroscopic observable. So the coherence domains can explain the effectiveness of ultra-weak and precise information managed by the organism, and the transition from the quantum to the macroscopic level.

But how do proteins manage information?

Popp has discovered that DNA emits biophotons [38], which are an ultra-weak cell radiation. The DNA then is not only the manager of the programmed cellular activities, but is also an emitter of electromagnetic waves that control the cellular mechanisms through continuous coupling reagents. DNA constructs and emits information. We can expect that proteins would do the same, even by their folding. At this point they function as a switch, and they will be able to start the enzymatic cascade, or the response of the network, even at a distance. In addition, the electromagnetic field can be transmitted by the coherence domains, and allows the transmission of a message coming from the molecules. This allows 
them a long haul attraction, if the emission spectrum is identical. It creates a field of coresonant molecules, which in turn will resonate with other adjacent molecules, and then with their groupings. This permits minimum changes made by a wave ultra-weak to become macroscopic and be observable.

The fascinating thing is the informed dissipative structure and water-protein-other solutes, which effectively makes the water a switch that can turn on or off some vital functions through the structural coupling. This could also explain the origin of life through water.

\section{The informational point of view}

Now I analyze everything from the point of view of the information. Relatively to information, three types of systems are defined [20]:

- Information-conserving systems. The distribution of their possible equilibrium in the phase space is conserved.

- Information-compressing systems. They have a finite number of possible equilibrium states and follow the second principle of thermodynamics.

- Information-amplifying systems. They are more complex, not linear. They are open systems, with an infinite number of possible equilibrium states. We are part of this category. All three types of systems are deterministic, though in the latter case we can predict what will happen at the local level, but not at the global one. My hope is to get step-by-step by adding computational information. Currently the non-predictability is due mostly to our observer's limits.

I formulated this hypothesis that I illustrate with the formula below: each entity has an "information ratio", a information coefficient that acts at a biological level, which is equal to:

$$
C i=\lim _{m \rightarrow 0} \frac{E}{m c^{2}}
$$

That is the information that leads comparing to its mass. For example, the food has lower information coefficient than spices, because we need to ingest an higher mass of food than spices to have systemic effects.

According to another hypothesis I do, given the information we will have then a "magnitude" in the body expressed as the number of events that occur after it, which responds to this formula:

$$
M=\sum_{I=0}^{\infty} \frac{C i \cdot \log _{2} N \cdot K i \cdot U i \cdot K s}{\text { Entropy }}
$$

Where:

- $\mathrm{K} i$ is the number of node connections 
- $\mathrm{N}$ is the number of affected nodes

- $\mathrm{C} i$ is the coefficient information

- $\mathrm{U} i$ is the value associated with the node

- Ks is the coefficient of susceptibility of the organism to information

Regarding the last point, it is necessary to consider also the susceptibility of the system, which is not only given by the number of nodes reached by information. Let's think of an allergy sufferer. With the same number of nodes initially involved, the susceptibility to a given substance is greater than for other people, and consequently also the magnitude of the response.

In addition, the more the points touched by information will be, and much heavier and more connected they will be, the greater the effect of information. This is also directly proportional to the systemic susceptibility, which is related to the coherence and to the afferent domain of body language by information. Similarly, the more the system will be entropic, the fewer events it will allow. A more entropic system is a disordered system, less harmonious, and less susceptible to knowledge and change. It always knows less, is less and less susceptible, it is less and less changeable. It is not influenced as before. This deterioration continues until death.

The centrality of a node is defined by Bianconi and Barabasi [39]:

$$
b_{i}=\sum_{j, w} \frac{g_{j i w}}{g_{j w}}
$$

Where $\mathrm{G}_{j i w}$ is the number of the shortest routes from $j$ to $w$ passing through $i$ (the node), and $\mathrm{G}_{j w}$ is the number of the shortest routes from $j$ to $w$ without passing through $i$.

The centrality of the node is given by the ratio between the number of connections from a point to a point $w j$ that pass through the node and those linking the same two points without question him.

Bianconi and Barabasi have compared their model to the evolution of a Bose gas, proposing that the energy of each node is equal to:

$$
\varepsilon_{i}=-\frac{1}{\beta} \log \left(U_{i}\right)
$$

where:

$$
\beta=\frac{1}{T}
$$

That is, the energy of a node is proportional to the temperature and to the weight logarithm, or fitness value of the node. The nodes with greater weight acquire more and more links, connections, at a faster rate. Then, at the approach of the thermodynamic limit, the connectivity decreases towards zero and shows the hierarchy of hubs, that is of larger 
groups of nodes, surrounded by other less connected nodes [40].

These activities have been modeled to explain networks of people who produce, disseminate and exchange ideas, but are also compatible with the network organization of our system.

In particular, the response always activates one or more central nodes, which increase the connections with the others, and that implements the answer to a typical and complex cascade. The response networks are then created, which give rise to observable.

These activities do not take place only in top-down way, i.e. in a hierarchical order from top to bottom, but may occur in particular in the opposite direction, the bottom up [41].

It is important to define the type of information I am talking about. In a quantum system, the information is a sequence of qbit, which have been superimposed of 0 and 1 . When we measure it, we operate a reduction of the state vector, $\mathrm{R}$. We get a 0 or a 1 , which, however, are derived directly from our measurement. It is said that the bits we observed already existed before. So Shannon's information is not useful in this case [42], partly because the quantum system is not closed, as it is open to the viewer. Moreover, as we have already seen, with each measurement we get decoherence, states are no longer overlapping, and then we lose information.

The information that interests us is that of Fisher, which is connected to the quantum potential. In fact, the quantum potential emerges as a result of balance classic under the constraint of a minimal presence of Fisher information [43]. The Fisher information can be interpreted as the information that an observable random variable $\mathrm{X}$ carries about an unobservable parameter $\theta$, which determine the probability distribution X. This statistical measure has sparked interest in relation to the study of the distributions of observables a quantum system [44]. Interesting studies were published; an attempt was made to connect Fisher's information with the specific structural aspects of quantum mechanics [45-49]. In this way this information is treated as a statistical indicator of the relationship between the classical information; also connecting the observed statistical system and its overall geometry.

When we apply this choice, avoiding the use of Shannon information, we know that the total information of a system can be encoded in a single observable (for example, the quantum potential) or may be partially encoded in all the complementary partial observable.

MacLennan [50, 51] wrote two enlightening articles about information and its computation in natural systems. The computation to universal restraint today, certainly the best known, is that of Turing, in which a machine processes numeric data and gives precise answers. Natural computation instead occurs in nature or is inspired by it. Examples of natural computing are the processes of the immune system, the brain computation, the selective evolution.

The natural computing tries to explain the processing of information by the natural and artificial systems, and therefore takes into account their characteristics, which are:

- Rapid, real-time response. 
- Flexibility in response, even in the presence of novelty.

- Adapting to new information and the environment, immediate and also gradually over time.

- Tolerance of noises (i.e. interference), errors, damage or fallacies.

- The robustness against environmental hazards, and also the damage.

- At the same time, the need to commit only small errors, the results of which are limited.

- I would add, the ability to self-repair.

For these reasons an analog model is advanced, in which the variations are small in magnitude as indicated and the variables are continuous.

The natural system is modeled through continuous representation spaces, which present uncertainty and indeterminacy: for each potentially correct representation there are others similar.

The other big difference between the natural computing and that of Turing is this: the first ends only when the system dies. The natural computing systems do not solve problems in the strict sense, but work on response and adaptation strategies to stimuli [52].

The information is encoded by continuous variables and phases of pulses. Mac Lennan proposes the use of Hilbert spaces, and suggests that the information representation is a field, i.e. an extended spatial continuum in which each change (that is, with the addition of information) following new snapshots configurations, that govern state changes continue. This dynamic is not necessarily deterministic. If these phenomena occur in a nondeterministic way, consider once again the probability of all the trajectories of the density function. This is continuous.

The space and the topology of the field are important both for the information they represent and the interactions that they allow. In the informational field move two types of information: the semantics (meaning) and the pragmatic (procedures). The signal shape in driving input determines the type of action; the magnitude gives the exact data. MacLennan makes also an important analogy: just as in the quantum field particles are excitations of the field, so in the mind computation reveals discrete symbols by continuous neurological processes.

\section{From the quantum to the classical level - the macroscopic observation}

At this point, the logical question is: how do we keep at least part of what happens at the level that we described? The collapse of the wave function, the $R$ reduction of states of quantum superposition causes the observable, which is the symptom or the potentially recognizable external manifestation.

If we want a physical parameter that gives us an overview we could observe the spectrum of bio photons emitted from the body at a given instant. This would be described by a wave 
function. In fact, the more the body is healthy and the energy is high, the more the spectrum is towards the blue and the purple, while with the entropy increasing, the decrease of energy and the presence of diseases the spectrum shifts to the red. This is also a consequence of Planck's formula $\mathrm{E}=\mathrm{hv}$. I may at this point also analyze the spectrum of information that we are giving out, to ensure that it is as similar as possible to that of the organism, so that the two waves are annihilated by subtraction. But, as long as the search does not progress in this, we can use another method, the integrated diagnostic tests we have available.

The most direct, simple, accurate, unique and individualized approach is to observe the symptoms that the patient reports. The symptoms, if observed without prejudice and recorded in their entirety, allow to qualitatively observe the systemic changes. I am speaking of specific, precise symptoms, such as: "pressure sore at the top of the right shoulder, which worsens to the touch; breathing deeply an obtuse pain, which extends behind and outside, inside the shoulder joint", or "stabbing pain in the umbilical region, in a small point, which forces him to bend and bow forward, aggravated to the highest pitch by lifting something, for 18 hours", or "falls asleep tired, and sleeps, though with difficulty, but is awake long before you go back to sleep, even if in the morning you will not feel tired" [53].

It is clear that a symptom alone does not tell us anything: it will be the set of all the symptoms that will allow us to observe the underlying network, and see if there are logical connections, but not necessarily causal correlation. That is the distinction of accompanying symptoms of a disease or problem that indicates the type and mode of activation in the underlying networks for each individual patient. In this way we can also know exactly what is happening at the microscopic level, because the symptoms are a final summation of previous or ongoing events, and build the impression that quantum phenomena leave our classic real field.

It is only in this way, with the integration of information and more precise and observable at different levels, that an individualized medicine will be possible $[54,55]$.

\section{Conclusion}

I described the organism and its deepest part through the quantum field, explained its susceptibility through the coherence domains and Hopfield networks, its organization through the nodes and the management of information and internal changes. Another very important thing is the definition of the information, in particular what an effective remedy could be. I described it through a specific ultra-weak wave, that does not activate primarily the receptors, but modifies the quantum field and the coherence domains. These changes allow the secondary opening of the receptors and the consequent cascade of events, from which some macroscopic observable arise. I explained also how the system and the environment are coupled through the dynamic exchange of information and energy. I also 
analyzed the evolution of the system over time and the relationship between the observer and observed, with the strategic union of classical, quantum field and information physics. These findings open other doors for reading and see new realities, to understand otherwise inexplicable facts, to advance in our knowledge and in our practice.

The implications of this model are many and far-reaching: we can begin to study the physiology and pharmacology to a deeper level; we can definitely combine physics, mathematics, philosophy and medicine by raising the level of our discipline; we can figure out how to view the system as a whole; we can study the different systemic networks with a new conceptual framework, to understand which are the most important nodes and how to address them; we can begin to develop remedies that are similar to the operations of the system, which stimulate the healing process without blocking its answers. These must carry pure information. They do not need to act on the receptors: they must radically change the network response and help the whole body and its inner capacity of healing and reorganization.

We can also understand what the peculiarities of each system are, and develop a true individualized medicine, respectful of each individual person and as effective as possible. All without burdensome side effects, without the need to add a drug after another once the last administered has exhausted its effect, without the decline of the immune or cognitive response more and more.

Further studies are required to measure the systemic happenings at the quantum and coherence domains level. In particular this model has to be tested and consequently amplified. We need to deepen our mathematical and descriptive knowledge of the organism in order to measure the universal and individual responses to different types of information. This innovative vision allows the development of new therapeutic strategies and kind of drugs, of stimuli, that act in the way we presented. The more knowledge we acquire, the better we can act to help the healing process. 
Conflicts of Interest: I do not have any conflict of interest to declare.

Funding sources: This research did not receive any specific grant from funding agencies in the public, commercial, or not-for-profit sectors. 


\section{References:}

1. Diani S. Are Diseases the Best Possible Response of the Complex Living System to Stimuli? International Journal of History and Philosophy of Medicine 2018; 8: 10802.

2. Prigogine I. Time, Structure, and Fluctuations. Science 1978; 201, 777-785

3. Prigogine I, Stengers, I. La Nouvelle Alliance: Métamorphose de La Science. Gallimard

Editors, Paris, France, 1979

4. Hameroff SR, Craddock TJ, Tuszynski JA. Quantum effects in the understanding of consciousness. J. Integr. Neurosci, 2014 doi: 10.1142/S0219635214400093

5. Licata I. Why the Collective Behavior of Classic Neurons is so Well Approximated by a

Quantum Potential? Adv. Science, Engineering and Medicine, 2014; Volume 6, 712-714(3)

6. Licata I. Physics and Logical openness in Cognitive Models. Arxiv.

http://arxiv.org/pdf/nlin/0703066.pdf , 2007

7. Conte E, Licata I, Alelú-Paz R. A Quantum Neurological Model of PerceptionCognition and Awareness in Ambiguous Figures and the Case of the Dalmatian Dog. JBBS, 2015. doi:10.4236/jbbs.2015.512051

8. Freeman WJ, Vitiello G. The Dissipative Brain and Non-Equilibrium Thermodynamics. J.

Cosmol. 2011; 14, 4461-4468

9. Vitiello G. Dissipation and memory capacity in the quantum brain model. Int. J. Mod. Phys.

B, 1995 doi: 10.1142/S0217979295000380

10.Vitiello G. My double Unveiled: the dissipative quantum model of brain. John Benjamins

Publishing Company, Amsterdam, 2001

11. Maturana HR, Varela FJ. Autopoiesis and Cognition: The Realization of the Living. D.

Reidel Publishing Company, Dordrecht, Holland, 1980

12. Maturana HR, Varela FJ. The Tree of Knowledge: The Biological Roots of Human Understanding. Shambala, Boston (1987)

13. Hahnemann S. Organon dell'arte di guarire, VI Edizione. Edi-Lombardo, Roma, Italy, 2004

14. Tarlaci S. Quantum physics in living matter: from quantum biology to quantum neurobiology. Neuroquant. 2011; 9 (4), 692-701

15. Tarlaci S, Pregnolato M. Quantum neurophysics: From non-living matter to quantum neurobiology and psychopathology. Int. J. Psychophysiol. 2015; 103, 161-173

16. Singh TP: Possible role of gravity in collapse of the wave-function: a brief survey of some 
ideas. J. Phys. Conf. Ser. 2015; 626 no.1

17. von Lucadou W. Psyche und Chaos. Theorien der Parapsychologie. Frankfurt, Germany, 1995

18. Weizsäcker EU. Wiederaufnahme der begrifflichen Frage: Was ist information? Nova Acta

Leopold. 1972; 206, 536-555

19. Penrose R. On Gravity's role in Quantum State Reduction. General Relativity and Gravitation, 1996; 28, Issue 5, 581-600

20. Licata I. Emergence and Computation at the Edge of Classical and Quantum Systems. In: Licata I., Sakaji A. (eds.) Physics of Emergence and Organization, World Scientific, Singapore, 2007

21. Penrose R. The emperor's new mind. Oxford University Press, Oxford, 1989

22. Diósi L. Models for universal reduction of macroscopic quantum fluctuations. Phys. Rev. A

1989; 40, 1165-1174

23. Ghirardi GC, Rimini A, Weber T. Unified dynamics for microscopic and macroscopic

systems. Phys Rev D Part Fields 1986; Jul 15;34(2), 470-491

24. Pearle P. Combining stochastic dynamical state-vector reduction with spontaneous localization. Phys Rev A Gen Phys. 1989; Mar 1;39(5), 2277-2289

25. Longtin L, Mattuck RD. Relativistically Covariant Bohm-Bub Hidden-Variable Theory

for Spin Measurement of a Single Particle. Found. of Phys., 1984; 14, 8, 685-703

26. Károlyházy F, Frenkel A, Lukács B. On the Possible Role of Gravity on the Reduction of the Wave Function. In: Penrose, R., Isham, C.I. (eds.) Quantum Concepts in Space and Time, Oxford Univ. Press, Oxford, 1986

27. Bohm D. A Suggested Interpretation of the Quantum Theory in Terms of "Hidden Variables" I. Physical Review, 1952. doi:10.1103/PhysRev.85.166

28. Bohm D. A Suggested Interpretation of the Quantum Theory in Terms of "Hidden Variables" II. Physical Review, 1952. doi:10.1103/PhysRev.85.180.

29. Licata I. Transaction and Non Locality in Quantum Field Theory. EPJ Web of Conferences

70, 2014. doi:10.1051/epjconf/20147000039

30. Licata I. Effective Physical Processes and Active Information in Quantum Computing. Arxiv. https://arxiv.org/pdf/0705.1173.pdf , 2007

31. Logiurato F. L'interpretazione di Bohm della teoria quantistica. In: Fondamenti e interpretazioni della teoria quantistica. Università di Trento, Italy, 2004

32. Del Giudice E, Doglia S, Milani M. A collective dynamics in metabolically active cells.

Physica Scripta, 1982. doi: 10.1088/0031-8949/26/3/015 
33. Del Giudice E, Preparata G. Coherent dynamics in water as a possible explanation of

biological membranes formation. Journal of Biological Physics 1995; 20, Issue 1, 105-116

34. Del Giudice E, Doglia S, Milani M, Vitiello G. Structures, Correlations and Electromagnetic Interactions. In: living Matter: Theory and Applications in Coherence in Biology and Response to External Stimuli, pp. 49-64. H. Frohlich ed., Springer, Berlin, 1988

35. Hopfield JJ. Neural Networks and Physical Systems with Emergent Collective Computational Abilities. PNAS, 1982. doi:10.1073/pnas.79.8.2554

36. Hopfield JJ. Pattern recognition computation using action potential timing for stimulus

representation. Nature, 1995 doi:10.1038/376033a0

37. Bianconi G, Barabasi A-L. Bose-Einstein Condensation in complex networks. Phys. review letters, 2001. doi: 10.1103/PhysRevLett.86.5632

38. Popp FA. Properties of biophotons and their theoretical implications. Ind. J. Exp. Biol. 2003; 41, 391-402

39. Bianconi G, Barabasi A-L. Competition and multiscaling in evolving networks. Arxiv. arXiv:cond-mat/0011029v1, 2000

40. Huang K. Statistical mechanics. Wiley, Singapore, 1987

41. Lella L, Licata I. A new model for the organizational knowledge life circle. In: Processes of emergence of systems and systemic properties. Towards a general theory of emergence. (eds.) Minati G. \& Pessa E. Springer, Heidelberg, 2009

42. Brukner C, Zeilinger A. Conceptual Inadequacy of the Shannon Information in Quantum

Measurements. Arxiv. arXiv:quant-ph/0006087v3, 2002

43. Resconi, G., Licata, I., Fiscaletti, D. A Geometrical Manifold of Entropy for Fisher Information and Quantum Potential. Arxiv. https://arxiv.org/pdf/1110.5491.pdf, 2016

44. Licata I, Fiscaletti D. A Fisher-Bohm Geometry for Quantum Information. EJTP 2014; 11, No. 31, 71-88

45. Reginatto M. Derivation of the equations of nonrelativistic quantum mechanics using the

principle of minimum Fisher information. Phys. Rev. A. 1998; 58, 1775-1778

46. Shun-Long L. Fisher Information of Wavefunctions: Classical and Quantum, Chinese Phys.

Lett. 2006; 23, 3127-3130

47. Hall JWM. Quantum Properties of Classical Fisher Information. Phys. Rev. A, 2000 doi:

10.1103/PhysRevA.62.012107

48. Garbaczewski P. Random Dynamics, Entropy Production and Fisher Information. Acta Phys. Pol. B 2003; 34, 3555-3568 
49. Luati A. Maximum Fisher information in mixed state quantum systems. Ann. Statistics, 2004; 32, 1770-1779

50. MacLennan, BJ. Natural computation and non-Turing models of computation. Theoretical

computer science 2004; 317, 115-145

51. MacLennan BJ. Field computation in natural and artificial intelligence. Information Sciences, 1999. doi:10.1016/S0020-0255(99)00053-5

52. Licata I. Mente e computazione. Systema Naturae 2003; 5, 237-306

53. Hahnemann, S.: Materia Medica Pura. Edi-Lombardo, Roma, Italy, 2000

54. Diani S, Lombardo C. The Paradigm-Shift toward the Next Generation of Drugs. Preprints 2020, 2020010040 doi: 10.20944/preprints202001.0040.v1.

55. Lella L, Licata I, Minati G, Pristipino C, De Belvis AG, Pastorino R. AI Models for the Personalized Medicine - Semantic Scholar https://pdfs.semanticscholar.org/2322/ d0dbf2d5f64f2af23c4bbcafc568e8126d3e.pdf 